\title{
Septic physeal separation of proximal femur in a newborn
}

\author{
Roberto Schiavon · Andrea Borgo • \\ Andrea Micaglio
}

Received: 6 June 2008/Accepted: 19 April 2009/Published online: 26 May 2009

(C) Springer-Verlag 2009

\begin{abstract}
In newborns physeal separations and septic osteomyelitis or arthritis are unusual, representing a problem in diagnosis and treatment. Therapy needs to be carried out soon in order to prevent anatomical and functional consequences. Association between septic event and physeal separation is rare. We report a 28-day-old female, admitted for elevated temperature, who underwent three nonorthopaedic surgical procedures before, and orthopaedic evaluation 8 days after admission. After an X-ray and an ultrasonography a septic arthritis with consequent hip dislocation was supposed. Only at the time of surgery a separation between the epiphysio-trochanteric nuclei complex and the femoral shaft was observed, with clear hip joint. The interest in this case consists in the difficulty of the differential diagnosis at the first evaluation, the orthopaedic misdiagnosis based on the lack of complete preoperative imaging, and finally the long-term excellent result after a prompt surgical treatment.
\end{abstract}

Keywords Physeal fracture - Septic arthritis .

Diagnostic errors

\section{Introduction}

Physeal separations in newborns represents a problem from both the diagnostic and treatment point of view. In

R. Schiavon · A. Micaglio

Orthopaedic and Traumatology Unit,

Vicenza General Hospital, Vicenza, Italy

\section{A. Borgo $(\square)$}

UO Ortopedia e Traumatologia, Azienda Ospedaliera di Padova, Università degli Studi di Padova, Via Giustiniani 2,

35100 Padua, Italy

e-mail: andrea-borgo@libero.it developed countries, neonatal septic osteomyelitis and septic arthritis are uncommon and represent a problem with respect to the differential diagnosis among several other disease, that often are not strictly restricted in the orthopaedic and traumatology area and frequently needing all diagnostic resources to obtain the correct diagnosis in order to get the best knowledge of the actual condition and to avoid an unsuitable treatment or an unexpected anatomical situation in case of surgical exposition. Therapy needs to be initiated as soon as possible in order to prevent the potentially adverse anatomic and functional consequences $[1,2]$. Association between a septic event and physeal separation is extremely rare.

In order to illustrate the difficulties associated with a proper diagnosis and management, we report on a case of complete septic physeal separation in a newborn.

\section{Case report}

The patient's parents provided consent to the publication of this case report. A 28-day-old Caucasian newborn was brought to the paediatric emergency department for symptoms involving 2 days of elevated temperature. The infant was born by normal spontaneous vaginal delivery (NSVD), her mother received normal prenatal care with no evidence of any particular problems, and the pregnancy was uncomplicated. At the time of admission, symptoms included fever, chills and irritability. With the symptomatology suggestive of an abdominal problem, she was evaluated by the paediatric surgeon who reduced an inguinal hernia and admitted the infant to the paediatric surgery department for observation.

The following day the patient's clinical conditions did not improve, high fever and irritability persisted. 


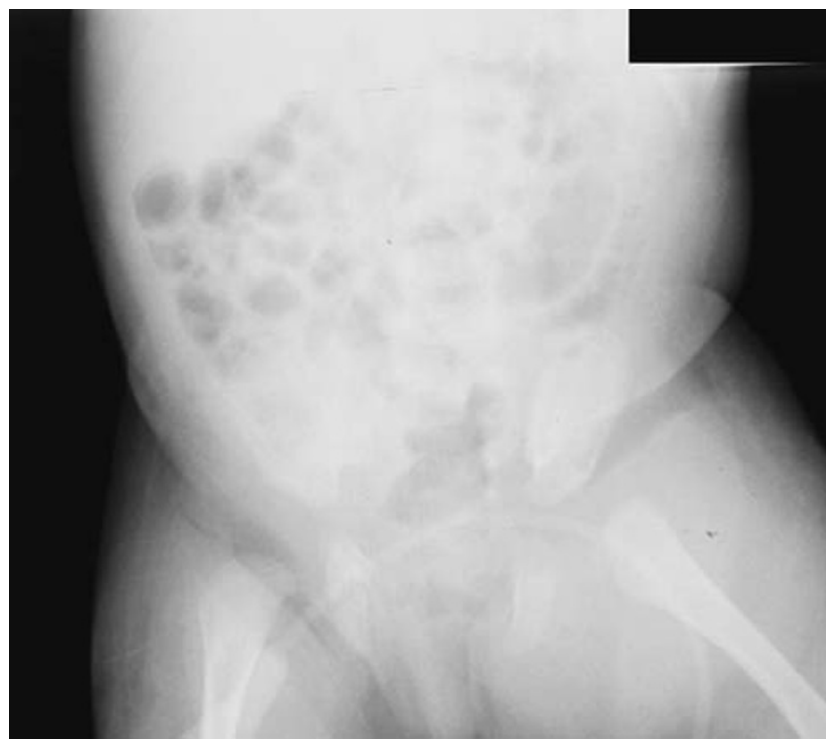

Fig. 1 Radiograph 3 days after admission: distension of the bowel loops is evident; note the flexion and rotation of the inferior left leg

A complete blood count (CBC) with differential showed leukocytosis with a neutrophylic predominance. Clinical conditions were still suggestive of an abdominal problem. An anteroposterior direct abdominal X-ray evidenced bowel loop distension (Fig. 1). Three days after admission the clinical condition remained unchanged: a diagnosis of intestinal occlusion was made and an explorative laparoscopy was performed but no evidence of an intestinal problem was present. During the same surgical session a bilateral open inguinal hernia repair was performed with bilateral reconstruction of the inguinal canal.

Two days postoperatively conditions were unchanged while a swelling of left hip and thigh region became noticeable. The skin was red and warm to the touch and the left leg was flexed and rotated. At this time a hypothesis of liquid collection in the hip-thigh region was formulated by the paediatric surgeon and needle drainage under ultrasonography was performed with evidence of pus presence which was immediately sent to the microbiological department for culturing. Only at this time was broad-spectrum antibiotic parenteral therapy started (ceftazidime $200 \times 2 \mathrm{mg}$, amikacina $40 \times 2 \mathrm{mg}$, metronidazole $60 \times 2 \mathrm{mg}$ ). Immediately after needle drainage and with the start of antibiotic therapy, status improved but without complete recovery.

An orthopaedic evaluation was requested only 8 days after admission. The persistence of a septic condition and the warm and reddened skin in the left hip region were suggestive of septic arthritis of the hip. New X-ray and a new ultrasonography studies were performed with a correct position of the pelvis.

The X-ray study with the correct position of the pelvis demonstrated the dislocation of the femoral head (Fig. 2),

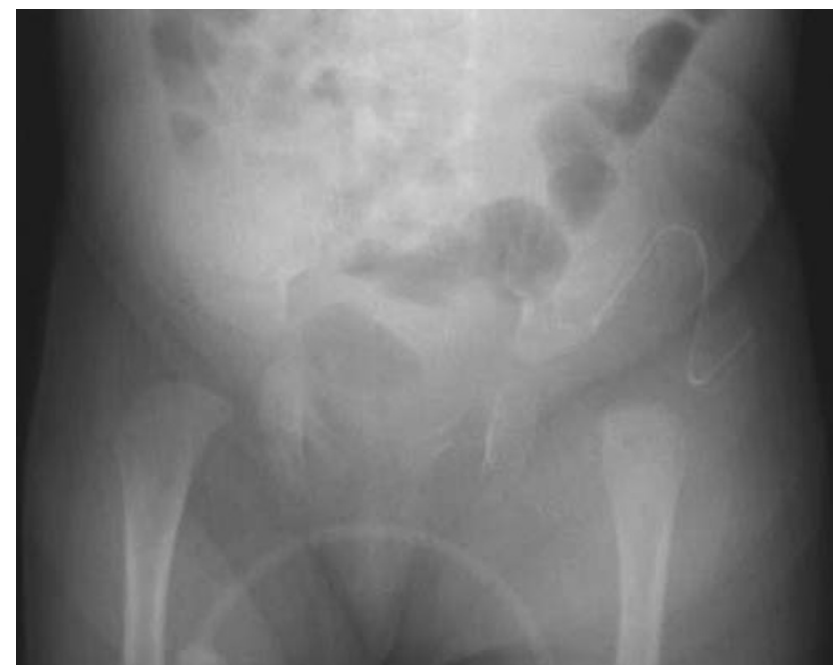

Fig. 2 Second radiograph: while performing that new radiograph, the proper pelvis position was held by an orthopaedic surgeon and it is possible to note a dislocation of the femoral head

while the ultrasonography findings revealed the presence of parostal proximal femoral shaft liquid collection and an undefined morphology of the hip. No more imaging was thought to be required and a diagnosis of septic hip arthritis with consequent hip dislocation was formulated (Fig. 3). Surgical open drainage was indicated. At that time, the first results from the microbiology culture became available, with evidence of growth of Gram-positive cocci (Staphylococcus aureus became evident 2 days later). Parenteral antibiotic therapy was modified with the suspension of ceftazidime and metronidazole; only amikacin was continued and cefotaxime was added.

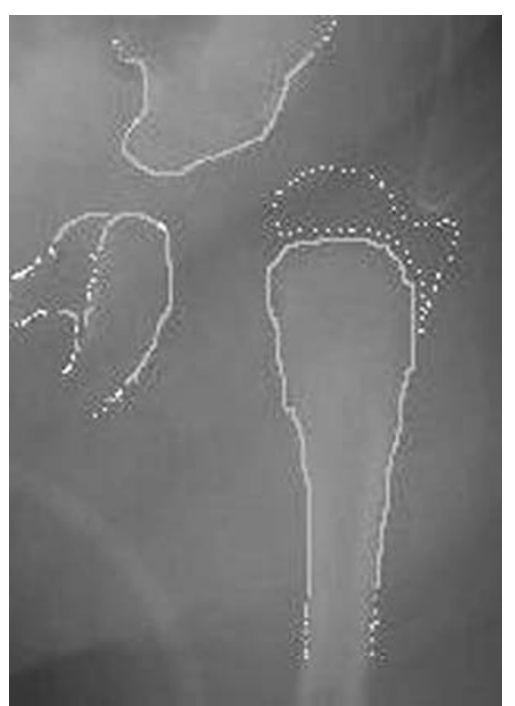

Fig. 3 After orthopaedic evaluation and new imaging in correct position a diagnosis of septic arthritis with consequent hip dislocation was formulated (as represented) and surgical open drainage was indicated. Imaging was thought to be sufficient 
Ten days after admission the infant underwent open surgical drainage by an orthopaedic team of the presumed hip joint septic arthritis by an anterior approach (SmithPetersen approach). Actually, only at time of surgery was it possible to observe a physeal separation between the epiphysio-trochanteric nuclei complex and the femoral shaft, with purulent liquid collection, but the hip joint plugged by the femoral head and the epiphysio-trochanteric growing nucleus was not involved in the septic process (Fig. 4).
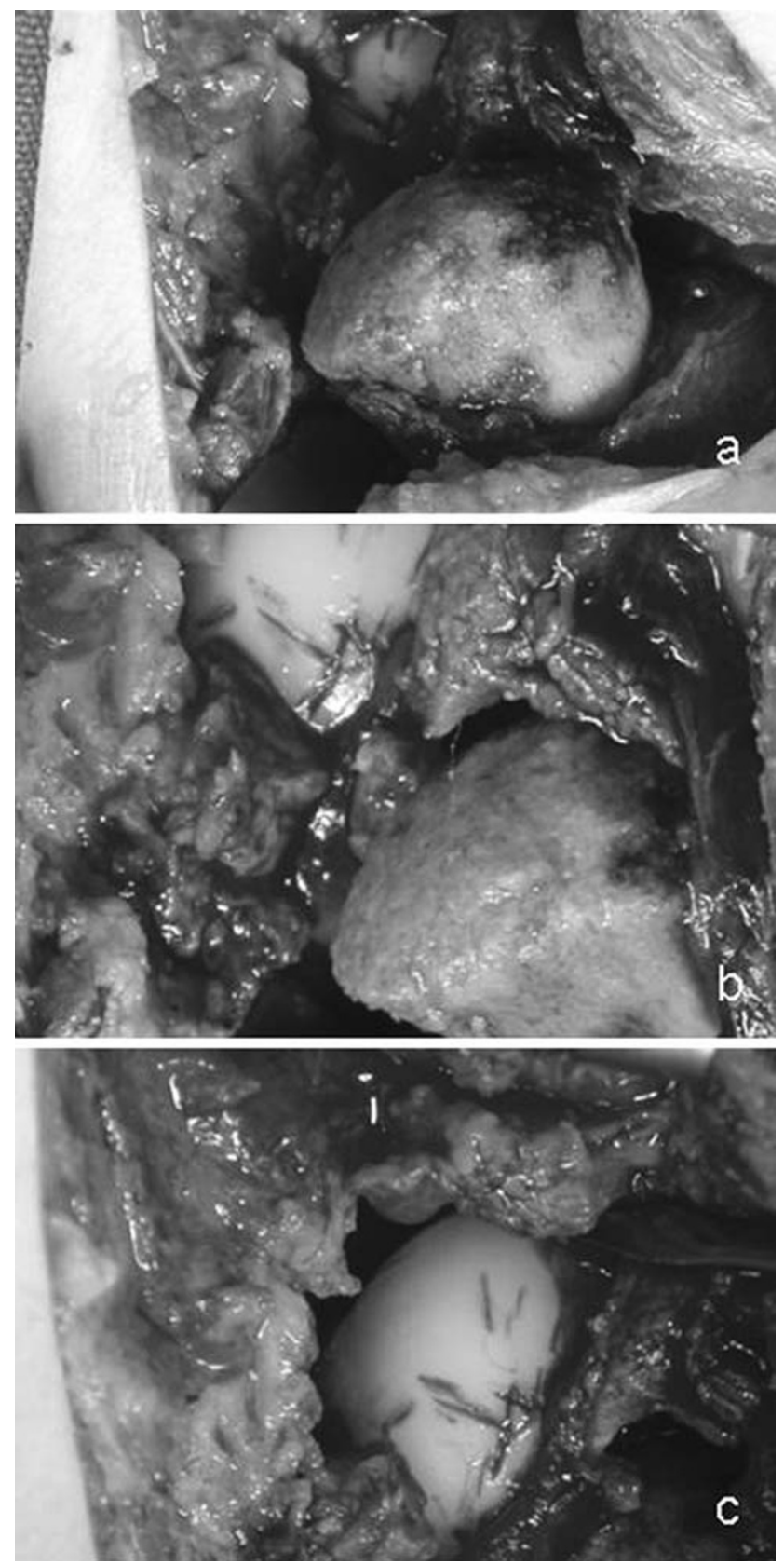

Fig. 4 Intraoperative finding: physeal separation between the epiphysio-trochanteric nuclei complex and the femoral shaft. (a) Hip joint was plugged by the femoral head and (b) the growing nucleus was not involved in the septic process (c)
After accurate draining and washing of the purulent fluid collection, the hip joint was opened by an anterior approach and the absence of pus presence inside the joint was confirmed. Only at this time was it possible to make the proper diagnosis of septic physeal separation between the epiphysio-trochanteric nuclei complex and the shaft of the proximal femur with clear hip joint (Fig. 5).

The epiphysio-trochanteric complex was gently reduced back to the anatomic position and stabilized with Kirschner wires. One suction drain was positioned inside the joint and another next to the proximal femur. After surgery, immobilization was achieved with plaster spica (Fig. 6).

Immediately after surgery the clinical conditions of the patient improved, with complete normalization of body temperature and absence of irritability. Parenteral antibiotic therapy was again modified (netilmicin $8 \times 3 \mathrm{mg}$, and oxacillin $200 \times 2 \mathrm{mg}$ ). Parenteral antibiotic therapy was continued until the second postoperative week.
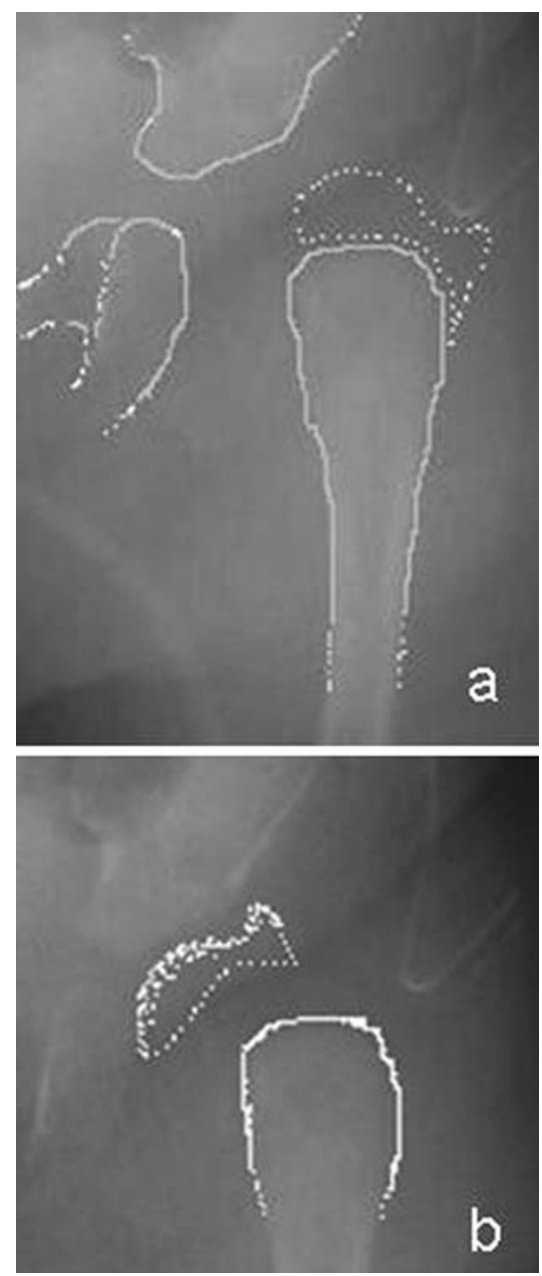

Fig. 5 Only at the time of surgery was it possible to achieve the correct diagnosis. We were not in presence of a septic hip arthritis with consequent hip dislocation as thought (a), but the correct diagnosis was septic physeal separation between the epiphysiotrochanteric nuclei complex and the femoral shaft (b) 

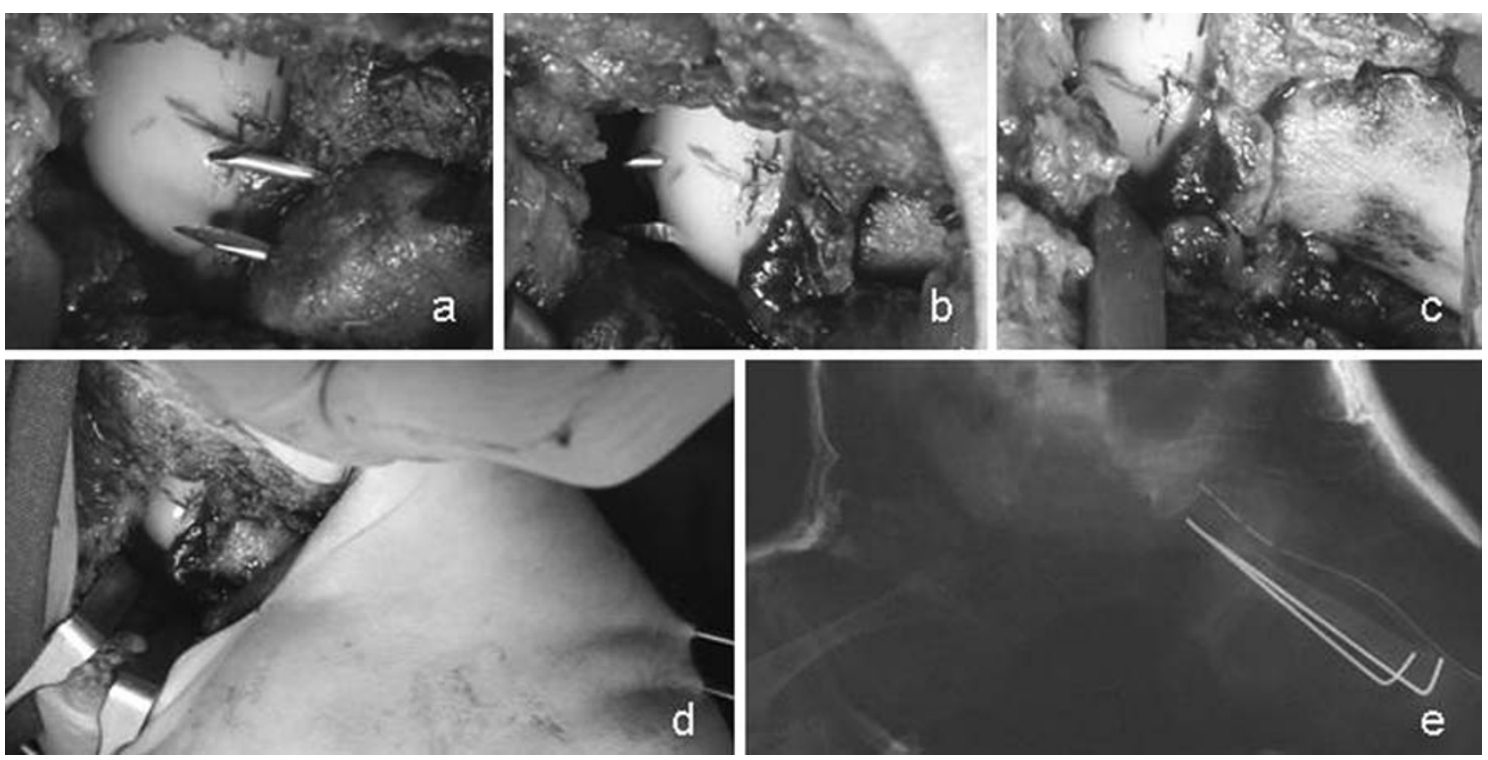

Fig. 6 Surgical procedure: the epiphysio-trochanteric nuclei complex was gently reduced back to the anatomic position and stabilization with Kirschner wires was obtained $(\mathbf{a}, \mathbf{b}, \mathbf{c}, \mathbf{d})$. After surgery, immobilization with plaster spica was provided (e)

Postoperative X-ray evaluation showed good alignment between the femoral shaft and the epiphysio-trochanteric nucleus, with a perfect position of the femoral head inside the acetabulum.

The infant was discharged 2 weeks following the last surgical procedure and continued with antibiotic therapy (riphampicine $25 \mathrm{mg} 1 \mathrm{tab}$ bis in die for 3 weeks).

Spica cast immobilization and $\mathrm{K}$-wires were maintained for 4 weeks. The patient followed-up at 2.5, 6, and
16.5 months, with progressive recovery of range of motion and good radiologic findings. At 4 years of age, her last follow-up showed that the hip joints were completely symmetric on X-ray imaging and equal in range of motion; there was no limb-length discrepancy or deformity, the child did not report any pain and was perfectly able to perform her daily activities including unlimited walking, running, and squatting (Fig. 7).
Fig. 7 Follow-up radiographs at 2.5 months (a), 6 months (b), and 16.5 months (c) and 4 years (d). Note the symmetry on $\mathrm{X}$-ray of the two hips. When last reviewed at 4 years, range of motion was complete and symmetric, there was no limblength discrepancy or deformity, the child did not complain pain and was perfectly able to perform her daily activity including unlimited walking, running, and squatting
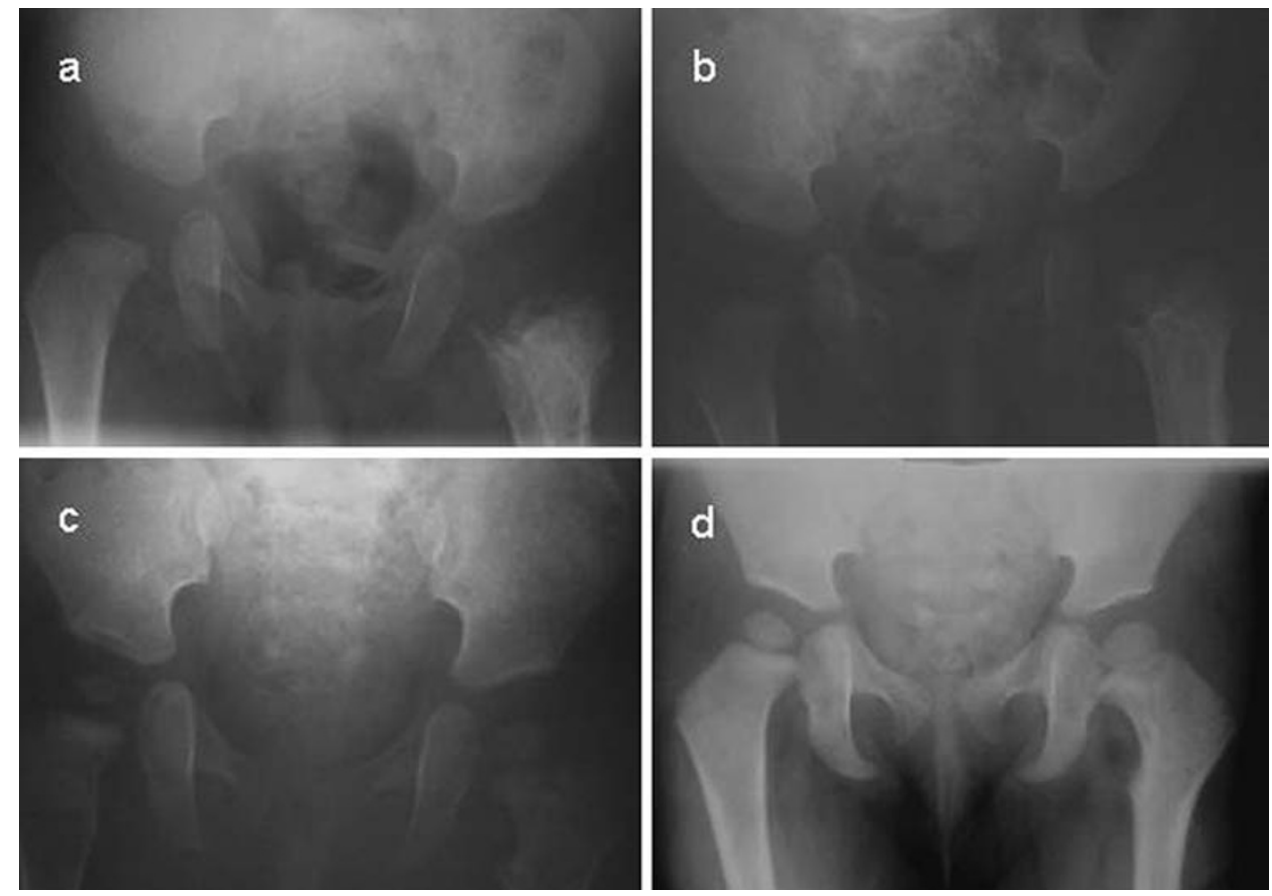


\section{Discussion}

Physeal separations in neonates occur rarely [3-7] and femoral involvement in physeal separation may occur either proximally or distally [3]. Concomitant physeal fracture and infection are very uncommon [3], and a complete separation of an epiphysis is described as a rare complication of neonatal osteomyelitis or septic arthritis [8]. The literature appears to indicate the hip joint as the most common site of epiphyseal slip as well as the most common site involved in a neonatal septic process [8].

Because of some anatomic and histological characteristics (large sinusoidal channel, loose metaphyseal cancellous structure, thin cortex, and loosely attached periosteum) typical of the neonatal age, the juxtaepiphyseal intraosseus pus can easily reach the subperiosteal space and affect the local soft tissue and joint. The confinement of bone destruction to the juxtaepiphyseal area with early cortical perforation and stripping of the periostium appears to have the capacity to induce a mechanism which leads to the loosening of the epiphysis. This is the process that leads to these two possible complications (septic arthritis and physeal separation) unique to the neonatal age [8-10].

Today, in developed countries, septic arthritis and acute osteomyelitis are quite uncommon in the very first days of life. Chorioamnionitis, prolonged membranes rupture, maternal chills or fever are considered risk factors for the child's septic events. Macrosomia, breech presentation, difficult or precipitous vaginal delivery, and a caesarean section could predispose to birth trauma [3]. Scurvy should always be considered in the event of a neonatal fracture when the history is negative for trauma [11]. Nonaccidental injury should be considered, particularly when other signs of violence are present.

In our case maternal risk factors for neonatal septic events were absent and delivery was uneventful and nontraumatic. No evidence of metabolic disease or child abuse was present.

Recognition of a physeal separation in a newborn is difficult, whether secondary to trauma or following a septic event. Physical findings of septic arthritis or osteomyelitis could be present, but multiple clinical evaluations could still be nonconclusive due to the patient's early age and to the difficulty involved in reaching a timely and accurate diagnosis [3].

In our case the newborn was admitted with a nonspecific symptomatology involving fever, chills, and irritability. No local sign suggestive of a hip infection was present. When first evaluated by the paediatric surgeon she was thought to have an abdominal problem. A hypothesis of fluid effusion was formulated only after considering the failure of the prescribed medical therapy, the negative findings obtained from an explorative laparoscopy, and the appearance of localized cutaneous redness and swelling. This diagnosis was later confirmed by a needle percutaneous drainage which evidenced the presence of purulent material. Only at this time was parenteral broad-spectrum antibiotic therapy started and, 8 days after admission, was an orthopaedic surgeon consulted and the diagnostic hypothesis of a septic arthritis formulated.

In newborns, standard radiographs may not identify the epiphysis because of the lack of ossification of the growing nucleus, and even if the ossification center is present, its minute dimension could be equivocal in plain radiographs which would not be able to detect a displacement. Only if the fracture involves part of the metaphysis may a tiny bone fragment separation appear [8]. A good view of the hips requires correct positioning of the pelvic ring, which can only be achieved when the lower extremities are firmly held in the appropriate position.

Our patient underwent an initial radiology study (X-ray) that in reality was aimed at evaluating the abdomen but, due to the small size of the child, was able to show a significant section of the two lower extremities. This first exam was thought to be normal. When the clinical condition changed and the hypothesis of a septic arthritis became evident, a second X-ray was performed. This time the child's legs were strongly held by the orthopaedic surgeon in the correct position and it was possible to notice the hip displacement. At this time, a review of and comparison with the initial films allowed us to formulate some doubt regarding the affected hip but the findings remained inconclusive due to the incorrect positioning of the lower extremities which disallowed an accurate clinical evaluation. This is an excellent example of the need to properly position the patient during the radiologic process. Ultrasonography is valid exam for the hip in newborn, able to show liquid presence inside the hip joint suggestive of septic arthritis. In our case ultrasonography (performed only when the local condition suggested a hip involvement), was able to demonstrate the presence of a liquid collection from the iliac wing to the inguinal canal, as well as confirm that the liquid collection was strongly contiguous with the hip joint where the articular synovial space was significantly increased; these findings were suggestive of septic arthritis with an extra-articular liquid collection. A second ultrasonography was directly performed by the orthopaedic surgeon after the second X-ray was obtained, and particular attention was given to maintaining the correct position during the US scanning. Ultrasound findings showed periarticular and proximal parostal femoral shaft liquid collection while the morphology of the hip was undefined. On the base of the clinical condition and of the radiological findings, a hypothesis of septic hip arthritis with consequent hip dislocation was formulated and an open surgical drainage was performed. No more imaging was believed to be necessary. 
The lack of additional preoperative imaging limited the diagnostic hypothesis and led to a wrong preoperative planning. Only at the time of surgery was the correct diagnosis of physeal separation between the epiphysiotrochanteric nuclei complex and the femoral shaft formulated. The lesson learned is to assure that, when possible, complete and detailed preoperative imaging should be obtained in order to have a correct preoperative diagnosis fundamental in planning the appropriate surgical procedure. Literature reports computed tomography (CT) and magnetic resonance imaging (MRI) as useful imaging devices able to yield more detailed knowledge of the actual condition $[3,12,13]$. It is our opinion that a MRI study would have been preferable over a CT evaluation as it would have spared the young patient exposure to ionizing radiation and would have also furnished greater details of the area's soft tissues. MRI might have detected the correct cause of the problem, thus avoiding an incorrect diagnostic hypothesis that led to the abdominal surgical procedure.

Partial or complete slip of the proximal femoral epiphysis [14] has been reported as a complication of hip infection, while to the best of our knowledge, the complete separation of the epiphysio-trochanteric nuclei complex has been described only once [8].

While most partial or complete epiphyseal slip complicating hip infections reported in literature are late presenting, with a delayed or even missed diagnosis and an overall poor functional result with sequestration or destruction of the capital epiphysis, physeal pseudoarthrosis, ankylosis, great limb discrepancy, and need for surgical correction [1, 2, 8, 15-17], literature describes the extreme resistance to infections of the growing plate, underlining that an epiphyseal slipping complicating neonatal osteomyelitis appears to be consequence of a mechanical loosening and consequent blood supply compromise rather than infective destruction of the growing plate $[2,8]$. Consequently early correct diagnosis and early appropriate treatment appear to be correlated with good result $[3,12]$. Reduction and pinning with Kirschner wires is generally reported $[12,18]$.

In conclusion, despite the rarity of the association between musculoskeletal septic events and physeal separation, this potential diagnosis has to be considered in the neonatal age. Differential diagnosis at onset is not easy, especially when the newborn is at a very early stage and even the correct localization of the disease could be difficult to identify (our newborn underwent three surgical procedures before correct localization of the disease was established). Standard radiographs in newborns and in small children could be equivocal and in addition it is very important to hold a correct position while performing the exam. Ultrasonography is a valid exam to detect the presence of a fluid collection. In our case an additional complicating factor was constituted by the misdiagnosis which was based on the lack of a correct preoperative imaging (MRI was not performed and a diagnosis of septic arthritis was formulated). MRI appears to be the best diagnostic test which will allow for a detailed view of the joint(s) involved, thus allowing for a correct diagnosis and therapeutic approach. Finally we underline the excellent long-term results obtained after open drainage and closed reduction with pinning.

Conflict of interest statement The authors declare that they have no conflict of interest related to the publication of this manuscript.

\section{References}

1. Badgley CE, Yglesias L, Perham WS et al (1936) Study of the end results in 113 cases of septic hips. J Bone Joint Surg 18:1047-1061

2. Siffert RS (1957) The effect of juxta-epiphyseal pyogenic infection on epiphyseal growth. Clin Orthop 10:378-384

3. Mangurten HH, Puppala B, Knuth A (2005) Neonatal distal femoral physeal fracture requiring closed reduction an pinning. $\mathrm{J}$ Perinatol 25:216-219

4. Jain R, Binlski RJ (2001) Fracture of lower femural epiphysis in an infant at birth: a rare obstetrical injury. J Perinat 21:550552

5. Theodorou SD, Ierodiaconou MN, Mitsou A (1982) Obstetrical fracture-separation of the upper femoral epiphysis. Acta Orthop Scand 53:239-243

6. Ecklund K, Jaramillo D (2001) Imaging of growth plate disturbance in children. Radiol Clin North Am 39(4):823-841

7. Ecklund K, Jaramillo D (2002) Pattern of premature physeal arrest: MR imaging of 111 children. Am J Rad 1789:763-772

8. Arooj AJ, Johari AN (2000) Epiphyseal separation after neonatal osteomyelitis and septic arthritis. J Pediatr Orthop 20(4):554-559

9. Green WT (1935) Osteomyelitis in infancy. JAMA 105:18351839

10. Ogden JA (1979) Pediatric osteomyelitis and septic arthritis: the pathology of neonatal disease. Yale J Biol Med 52(5):423-448

11. Quiles M, Sanz TA (1988) Epiphyseal separation in scurvy. J Pediatr Orthop 8(2):223-225

12. Sferopoulos NK, Papavasiliou VA (1994) Proximal epiphyseal seoparation of the femur in the newborn: early ultrasonic diagnisis. Rev Chir Orthop Reparatrice Appar Mot 80(4):338-341

13. Azouz EM, Greenspan A, Marton D (1993) CT evaluation of primary epiphyseal bone abscesses. Skeletal Radiol 22(1):17-23

14. Graf R (1986) Sonographie der Sauglingshufte. Ferdinand Enkle Verlag, Stuttgart

15. Peters W, Irving J, Letts M (1992) Long-term effects of neonatal bone and joint infection on adjacent growth plates. J Pediatr Orthop 12(6):806-810

16. Dobbs MB, Sheridan JJ, Gordon JE et al (2003) Septic arthritis of the hip in infancy: long-term follow-up. J Pediatr Orthop 23(2):162-168

17. Riseborough EJ, Barret IR, Shapiro F (1983) Growth disturbances following distal femural physeal fracture fracture-separation. JBJS 65-A(7):354-356

18. Seller K, Wild A, Westhoff B et al (2006) Radiological evaluation of unstable (acute) slipped capital femoral epiphysis treated by pinning with Kirschner wires. J Pediatr Orthop B 15(5):328334 